\title{
Mechanisms of Nucleation and Stationary States of Electrochemically Generated Nanobubbles
}

\author{
Yamila A. Perez Sirkin, ${ }^{*}+{ }^{\dagger}$ Esteban D. Gadea, ${ }^{\dagger}$ Damian A. Scherlis, ${ }^{\dagger}$ and Valeria Molinero ${ }^{*},+\odot$ \\ ${ }^{\ddagger}$ Department of Chemistry, The University of Utah, 315 South 1400 East, Salt Lake City, Utah 84112-0850, United States \\ ${ }^{\dagger}$ Departamento de Química Inorgánica, Analítica y Química Física/INQUIMAE, Facultad de Ciencias Exactas y Naturales, \\ Universidad de Buenos Aires, Ciudad Universitaria, Pab. II, Buenos Aires C1428EHA, Argentina
}

Supporting Information

\begin{abstract}
Gas evolving reactions are ubiquitous in the operation of electrochemical devices. Recent studies of individual gas bubbles on nanoelectrodes have resulted in unprecedented control and insights on their formation. The experiments, however, lack the spatial resolution to elucidate the molecular pathway of nucleation of nanobubbles and their stationary size and shape. Here we use molecular simulations with an algorithm that mimics the electrochemical formation of gas, to investigate the mechanisms of nucleation of gas bubbles on nanoelectrodes, and characterize their stationary states. The simulations reproduce the experimental currents in the induction and stationary stages, and indicate that surface nanobubbles nucleate through a classical mechanism. We identify three distinct regimes for bubble nucleation, depending on the binding free energy per area of bubble to the electrode, $\Delta \gamma_{\text {bind }}$. If $\Delta \gamma_{\text {bind }}$ is negative, the nucleation is heterogeneous and the nanobubble remains bound to the electrode, resulting in a low-current stationary state. For very negative $\Delta \gamma$, the bubble fully wets the electrode, forming a one-layer-thick micropancake that nucleates without supersaturation. On the other hand, when $\Delta \gamma_{\text {bind }}>0$ the nanobubble nucleates homogeneously close to the electrode, but never attaches to it. We conclude that all surface nanobubbles must nucleate heterogeneously. The simulations reveal that the size and contact angle of stationary nanobubbles increase with the reaction driving force, although their residual current is invariant. The myriad of driven nonequilibrium stationary states with the same rate of production of gas, but distinct bubble properties, suggests that these dissipative systems have attractors that control the stationary current.
\end{abstract}

\section{INTRODUCTION}

Electrocatalytic generation of gas is key in processes that are important for energy and synthesis, ${ }^{1}$ such as water splitting, ${ }^{2,3}$ hydrogen evolution, ${ }^{4,5}$ regeneration of fuels by $\mathrm{CO}_{2}$ reduction, ${ }^{4}$ the electro-oxidation of methanol, ${ }^{6,7}$ and the industrial synthesis of chlorine. ${ }^{8}$ The formation of gas bubbles at the interface between electrode and electrolye has deleterious effects on the electrochemical reaction by, for example, decreasing the efficiency of the electrochemical processes by blocking the catalytic surfaces and increasing the reaction overpotential. ${ }^{9,10}$ A molecular understanding of the mechanisms and factors that control the formation, dissolution, and behavior of electrochemically generated gas bubbles is key to design efficient electrochemical processes and catalysts.

The push toward the development of nanocatalysts ${ }^{11-13}$ has made controlling and understanding electrochemically generated surface nanobubbles paramount. ${ }^{14}$ The electrochemical production, detection, and characterization of interfacial nanobubbles under controlled conditions have been investigated using various experimental approaches. ${ }^{5,15,16}$ The development of methods to produce and study single nanobubbles on nanoelectrodes ${ }^{15,17}$ has allowed unprecedented studies of the nucleation and stability of single nanobubbles of $\mathrm{H}_{2}, \mathrm{O}_{2}$, and $\mathrm{N}_{2}$ in nanodisk electrodes of radii ranging from 2.5 to $90 \mathrm{~nm} \cdot{ }^{1,5,15-29} \mathrm{In}$ all cases, the formation of nanobubbles was achieved after substantial gas supersaturation at the electrode surface. The formation of the nanobubble was evidenced by a dramatic drop in the current, caused by the blockage of the electrode surface by the bubble, to a nonzero residual value, $I_{r}$, which turned out to be essentially independent of the electrode potential. This residual current allows for the system to reach a dynamical steady-state, which results from the balance between the outward flux of gas diffusing away from the nanobubble into the solution, and an inward flux arising from the electrochemical reaction. Recent studies of Luo and co-workers have established that the supersaturation required for the nucleation of $\mathrm{H}_{2}$ nanobubbles is the same for $\mathrm{Pt}, \mathrm{Pd}$, and $\mathrm{Au}$ electrodes. ${ }^{28,29}$ Although the insensitivity of the critical supersaturation to the metal could be interpreted to arise from a homogeneous nucleation mechanism (as originally assumed for the nucleation of $\mathrm{H}_{2}$ nanobubbles on $\mathrm{Pt}^{17}$ ), recent analysis of hundreds of individual nucleation events indicate that $\mathrm{H}_{2}, \mathrm{~N}_{2}$, and $\mathrm{O}_{2}$ nanobubbles nucleate heterogeneously on Pt nanodisks. ${ }^{22,24,26}$ We note that even if the nucleation where homogeneous, it would occur very close to the surface, where the gas supersaturation is highest. It is not yet known whether electrochemically generated

Received: April 25, 2019

Published: June 13, 2019 
nanobubbles could nucleate through a homogeneous mechanism.

Electrochemical experiments do not yet have the spatial resolution to elucidate how and where gas bubbles nucleate on electrodes, their shape and size in the stationary states, or how to control and suppress their generation. ${ }^{1}$ Molecular simulations have the spatial resolution to address these questions. However, while several computational studies have been carried out to shed light on the unexpected stability and nucleation of nanobubbles, ${ }^{30-44}$ the nucleation and stationary states of electrochemically generated nanobubbles have never been studied by molecular simulations. In this work, we investigate the nucleation and stationary states of electrochemically generated nanobubbles, using molecular dynamics simulations with an algorithm that mimics the electrogeneration of gas at the electrode. The simulations reproduce the experimental results for the nucleation and stationary states of gas bubbles on Pt nanoelectrodes, demonstrate that surface nanobubbles must be nucleated heterogeneously, identify conditions that lead to nucleation of surface nanobubbles or one-layer-thick micropancakes, and reveal unanticipated changes in the size and shape of surface nanobubbles with the reaction driving force.

\section{RESULTS AND DISCUSSION}

\subsection{Simulations Reproduce the Experimental Cur-} rents and Stages in the Formation of Nanobubbles. We first investigate the process of nucleation and growth of gas nanobubbles on a $5 \mathrm{~nm}$ Pt-like diameter electrode exposed to water modeled with the $\mathrm{mW}$ model $^{45}$ and surrounded by an amorphous silica-like wall ${ }^{46}$ (Figure 1 and Methods). As

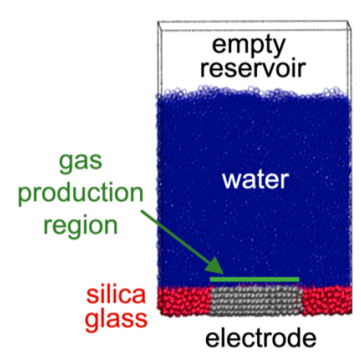

Figure 1. Schematic representation of the simulation cell. Particles that belong to the inert silica-like amorphous solid wall are shown in red, to the electrode are shown in gray, and to the water phase are shown in blue. The slab of vacuum prevents the increase in pressure as the gas is produced in the constant volume in the simulation cell.

experiments indicate that the phenomenology of nucleation of the gas bubble at the electrode is independent of the nature of the gas generated, ${ }^{20,25,26}$ we represent each gas molecule with a model of methane that reproduces the solubility of methane in water and its pressure dependence at $273 \mathrm{~K}^{47}$ as well as the liquid-gas surface tension, and structure of the watermethane interface. ${ }^{48}$ Although $\mathrm{CH}_{4}$ is not a gas typically generated in electrochemical experiments, its size, polarizability, equation of state, and solubility in water make it a good proxy for $\mathrm{N}_{2}{ }^{49}$

There are no gas molecules initially in the simulation cell (Figure 1). After some equilibration of the gas-free cell, we reset the time to zero and turn on the "electro-generation" of gas with a driving force for the chemical reaction given by the frequency $F$ of attempts at creating gas at the surface of the electrode (see Methods). Figure 2 shows the temporal

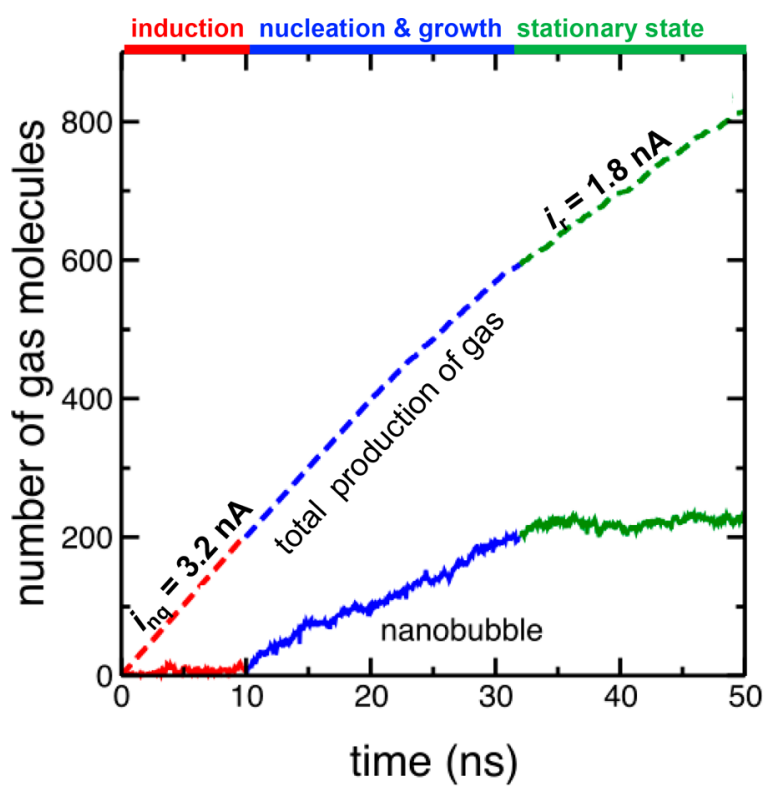

Figure 2. Evolution of the number of gas molecules with time. The dashed line represents the total number $N_{\mathrm{G}}$ of gas molecules in the simulation cell, while the continuous line shows the number $N_{\mathrm{B}}$ of gas molecules in the nanobubble, defined as the largest gas cluster. Red, blue, and green indicate the induction, nucleation and growth, and stationary states, respectively. Supporting Movies S1 and S2 show the formation of the gas bubble in this simulation from a top and side view with respect to the plane of the electrode. The current is proportional to $\mathrm{dN}_{\mathrm{G}} / \mathrm{d} t$. A current of $1 \mathrm{nA}$ corresponds to the flow of 6.25 electrons per nanosecond. If we assume that one electron is involved in the formation of the gas molecule, the current drops from $3.2 \mathrm{nA}$ (200 molecules in $10 \mathrm{~ns}$ ) in the induction stage to $1.8 \mathrm{nA}$ (200 molecules in $18 \mathrm{~ns}$ ) in the stationary state, in good agreement with the 2.9 and $1 \mathrm{nA}$ experimental currents just before nucleation and in the stationary states for generation of $\mathrm{H}_{2}$ on $5 \mathrm{~nm}$ diameter $\mathrm{Pt}$ nanoelectrodes. $^{18}$

evolution of the number of gas molecules in the cell for $F=$ $0.02 \mathrm{ps}^{-1}$ (i.e., one reaction attempt every $50 \mathrm{ps}$ ) and the size of the surface bubble. Each simulation has three well-defined stages as gas is produced at the electrode surface: (i) induction, (ii) nucleation and growth of the bubble, and (iii) dynamic stationary state (Figures 2 and 3 ).

Gas nuclei of subcritical size intermittently form at the electrode and dissolve into the solution during the induction stage that precedes the nucleation of the bubble (Movies S1 and S2). All gas creation attempts at this stage are successful, because the electrode is not blocked by gas. Hence, the current is constant in the induction state, with a value determined by the driving force $F$ for formation of gas and the area of the electrode. We denote this current, the largest one throughout the three stages, as the nonequilibrium current $i_{\text {nq }}$. If the rate of production of gas at the unblocked electrode is larger than the rate of diffusion of the gas molecules away into the solution, the concentration of gas over the electrode increases with time. The increase in supersaturation of the gas in the induction stage results in progressively more favorable conditions for bubble nucleation. At some point, the supersaturation of gas over the electrode is sufficiently large to nucleate the bubble in the short time scale of the simulations. The nucleation stage starts when a gas cluster that exceeds the critical size spontaneously forms and grows at the surface. The critical nucleus size in the simulations contains at least $\sim 30$ gas 
a) induction stage

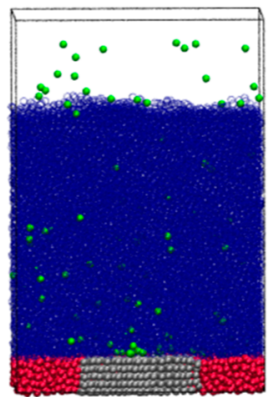

b) nucleation and growth

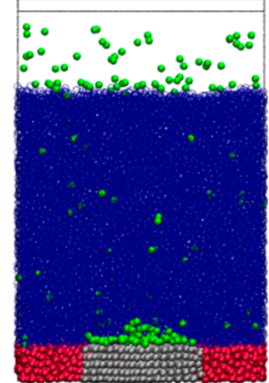

c) stationary state

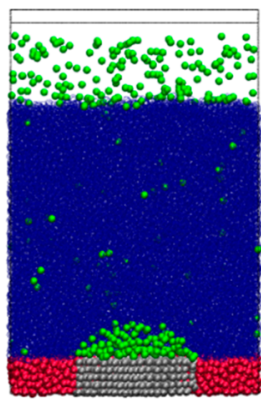

Figure 3. Snapshots of the three different stages in the formation of a surface nanobubble. (a) induction, (b) nucleation and growth, and (c) stationary state. This process is also shown in Supporting Movies S1 and S2. The particles of the silica-like amorphous solid wall, electrode, liquid water, and gas, are depicted in red, gray, blue, and green, respectively. The evolution of the size of the largest cluster along this simulation is shown in Figure 2. The cluster in panel b is already larger than the critical size. Note its low contact angle subtended between the bubble and the electrode, which agrees with the $\sim 20^{\circ}$ deduced from the rate of $\mathrm{N}_{2}$ bubble formation in experiments using Classical Nucleation Theory. ${ }^{26}$

molecules, in good agreement with the $\sim 40$ estimated for the critical $\mathrm{N}_{2}$ bubble through Classical Nucleation Theory (CNT) analysis of electrochemical experiments. ${ }^{26}$ The simulations indicate that the creation of the critical bubble nucleus does not involve the coalescence of multiple small nuclei (which we find to have a flickering lifetime at the electrode surface), but the creation of a single critical cluster that grows through addition of gas molecules, as predicted by CNT.

The critical cluster is dense, consistent with the high Laplace pressure expected for small bubbles. The average distance between gas molecules in the critical-sized bubble is $\sim 0.5 \mathrm{~nm}$, in excellent agreement with the $\sim 0.6 \mathrm{~nm}$ estimated for critical $\mathrm{N}_{2}$ bubbles from electrochemical experiments. ${ }^{26}$ Figure $2 \mathrm{~b}$ and Movies S1 and S2 show that the gas nuclei are quite flat. The contact angle $\theta_{\mathrm{c}}$ between the nascent bubble and the electrode (see Movie S2) is consistent with the $\sim 20^{\circ}$ deduced using $\mathrm{CNT}$ to interpret experimental rates of formation of $\mathrm{N}_{2}$ bubbles on $\mathrm{Pt}$ nanodisks. ${ }^{26}$ In agreement with the interpretation of electrochemical experiments, ${ }^{22,24,26}$ the molecular simulations show that the nucleation of the electrogenerated gas bubble is a classical heterogeneous process, catalyzed by the electrode.

The nucleation of the bubble is followed by its growth, which increasingly reduces the area available for the electrochemical reaction that produces new gas molecules. This results in a drop of the current until the system reaches a nonequilibrium stationary state in which the size of the bubble and the current do not change further because the number of gas molecules lost through the gas-water interface is compensated by the creation of gas molecules at the gaswater-electrode contact line. The residual current in the dynamic stationary state is $i_{\mathrm{r}} \sim 1.8 \mathrm{nA}$ assuming the gas forms through a one electron reaction. This value is comparable to the $\sim 1 \mathrm{nA}$ current determined in experiments of electrochemical generation of $\mathrm{H}_{2}$ nanobubbles on $\mathrm{Pt}$ nanodisks with the same diameter, $5 \mathrm{~nm} .{ }^{18}$ The stages and currents obtained in the molecular simulations are in remarkable agreement with those derived from electrochemical experiments.

The molecular simulations identify the region of the threephase water-electrode-gas contact line where the gas molecules are produced by the electrochemical reaction. This spatial resolution is not yet accessible through experiments. We find that thermal fluctuations of the three-phase line expose the boundary of the electrode to the solution, allowing the chemical reaction to proceed in a very narrow boundary of $\sim 1$ $\AA$ width (Figure 4). About the same width of reactive area was

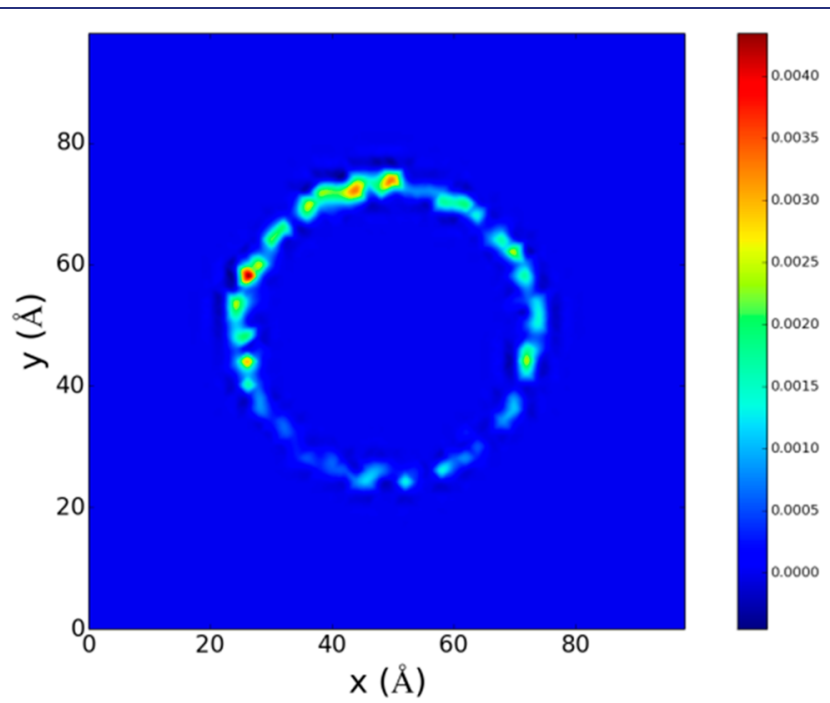

Figure 4. Gas formation events in the stationary state occur exclusively at the boundary of the electrode. The image shows the density of reaction events in the plane of the electrode averaged during $40 \mathrm{~ns}$ evolution of the stationary state of a $5 \mathrm{~nm}$ diameter electrode (centered at $x=50 \AA$ and $y=50 \AA$ ) in which the reaction proceeds with driving force $F=0.1 \mathrm{ps}^{-1}$. The coloring indicates the density of gas-creation events in units of $\AA^{-3}$. The bias for insertion in the upper left quadrant is related to the positioning of the bubble along the $40 \mathrm{~ns}$ sampling, and we expect will disappear with further sampling. The apparent hot spots (red areas) arise from nonuniformities in the silica glass as it meets the crystalline electrode. Simulations in which the inert wall is modeled with the same FCC lattice as the electrode result in a uniform distribution of gas creation events around the seamless rim of the reactive area under the same conditions of driving force and sampling time as the example of this figure.

deduced from finite element modeling of the stationary states of electrochemically generated $\mathrm{H}_{2}$ nanobubbles. ${ }^{15,23}$ Our analysis indicates that a narrow fluctuating region at the three-phase interface is the locus for all the current that keeps the nanobubble in a stationary state.

2.2. Size of the Stationary Nanobubble Depends on the Driving Force, Although Its Current Does Not. The electrochemical experiments indicate that the residual current is essentially independent of the applied potential. ${ }^{15,18,19,25}$ The frequency of attempts at creating gas molecules, $F$, plays the role of the reactive driving force in the simulations. While $F$ cannot be assumed to mimic the electrochemical potential, it plays the same role. Figure 5 shows the residual current $i_{r}$ as a function of $F$ in the simulations with the same $5 \mathrm{~nm}$ diameter electrode. The residual current is independent of the driving force, in agreement with the insensitivity of $i_{r}$ to the voltage in experiments. ${ }^{15,18}$ This agreement further validates that the simulation approach developed in this study captures the 


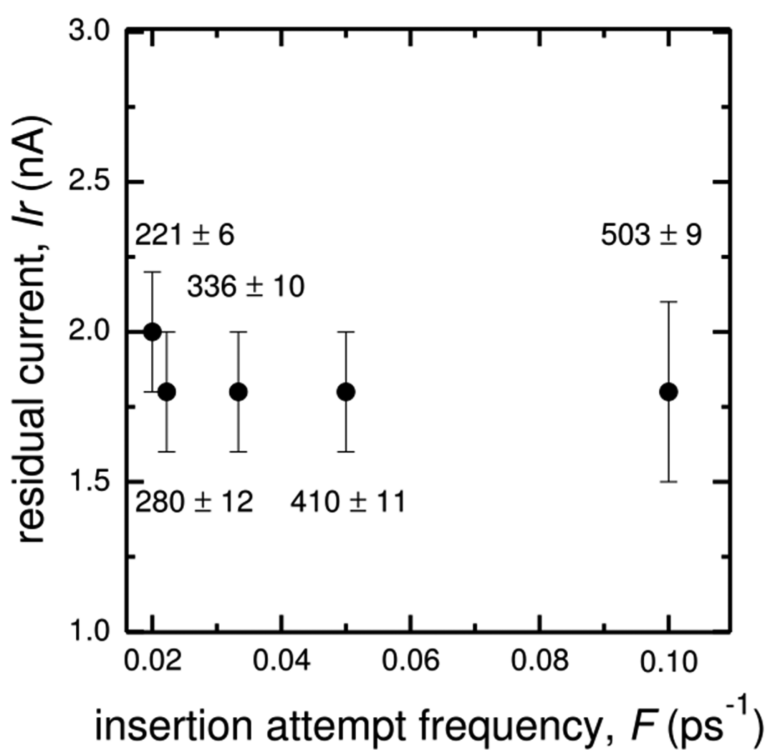

Figure 5. Residual steady-state current $i_{\mathrm{r}}$ as a function of the insertion attempt frequency $F$ for the $5 \mathrm{~nm}$ diameter electrode. The numbers next to the data-points indicate the mean number of molecules in the steady state nanobubbles for each condition. The interaction parameters are those of Table 1 of Methods.

physics of the feedback between reactivity and diffusion that controls the formation and stationary state of electrochemically generated nanobubbles.

There is a minimum value for the driving force $F$ below which the nucleation of the bubble does not occur in the simulations, as is also the case in the experiments. ${ }^{1}$ This threshold is determined by the balance between diffusion away of gas molecules through the bubble-solution interface and the generation rate of new molecules at the electrode surface: when the loss of molecules through diffusion is faster than the amount produced by the reaction, the gas supersaturation necessary to form the critical clusters cannot be reached. Not only is the bubble unable to nucleate when the driving force is too low, under those conditions the electrode cannot sustain a stationary state. The simulations show that an existing bubble dissolves when $F$ is set to a low value. Likewise, nanobubbles dissolve when the scanning direction of the potential is reversed after the bubble has been nucleated in experiments. ${ }^{15,20}$

As a dependence of the nanobubble size and shape on the driving force for the electrochemical reaction cannot be obtained from the experiments, it has been assumed that the size of stationary bubbles is, like the residual currents, invariant with the driving force. Our simulations reveal that this is not the case: the size of the stationary bubbles increases with the reaction driving force (Figure 6), although the current does not (Figure 5). The stationary bubble at the highest driving force in Figure 6 has over twice as many gas molecules than at the lowest $F$, despite having practically identical residual currents (Figure 5). This suggests that each nanoelectrode can produce an infinite set of stationary states with identical currents, but different sizes and internal pressures. More work is needed to elucidate the physical origin of the attractor that generates the manifold of bubble states with the same current.

Not only the size of the nanobubble increases with the driving force, the contact angle $\theta$ that the stationary bubbles make with the electrode also increases with $F$ (Figure 6). To

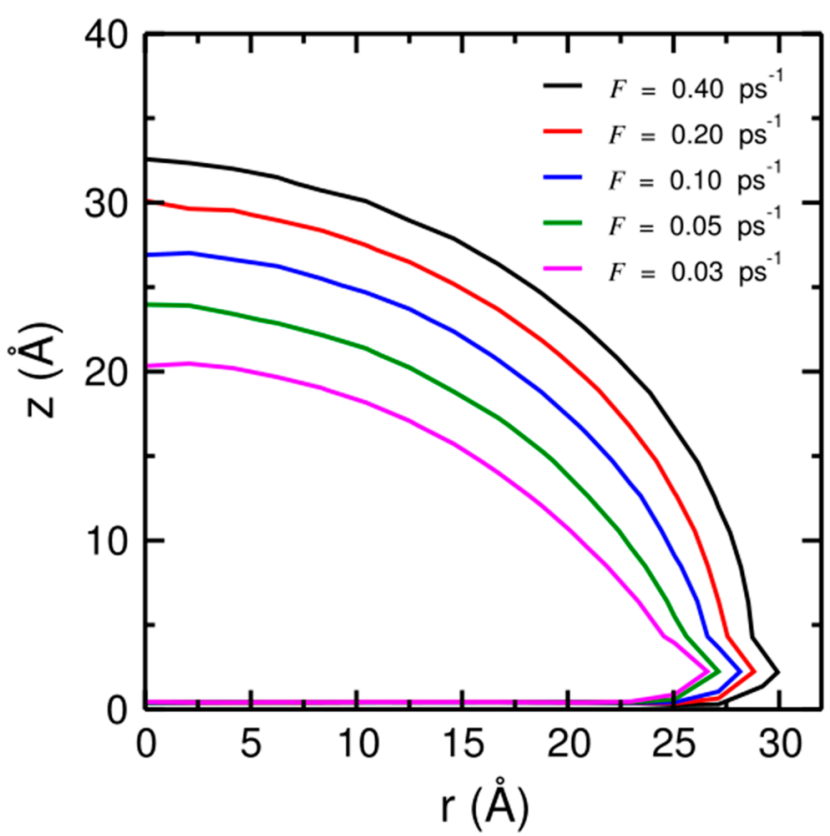

Figure 6. Size and contact angle of stationary nanobubbles increase with the reaction driving force. All the stationary bubbles evolved in the same $5 \mathrm{~nm}$ diameter electrode with the same interaction potential listed in Table 1 of Methods. The residual currents of all these bubbles are identical within the error bar (Figure 5). The height $z$ of the bubble is presented relative to the plane of the surface of the electrode; its radius $r$ from the center of the bubble.

understand whether the increase in the contact angle of the stationary nanobubble with driving force is due to nonequilibrium effects or can be ascribed to the difference in wetting properties of the electrode and the amorphous silica that surrounds it, we prepare a simulation cell in which the electrode and the wall have the same fcc structure of the Pt-like electrode and identical interactions with gas and with water (strength of the attraction $\varepsilon=0.45 \mathrm{kcal} \mathrm{mol}^{-1}$ ), but only a 2.5 $\mathrm{nm}$ radius region (the electrode) has the ability to produce gas at the surface. In that case the bubble nucleates on the electrode, but it is not pinned to it and it has a contact angle that seems to be independent of its size.

The constant angle of bubbles on electrodes that have the same wetting properties than the surrounding wall suggests that the size and contact angle of the bubble are equilibrium properties determined by the balance of the binding free energies of the bubble to the electrode and the surrounding amorphous silica surface. This conclusion is further supported by the similar evolution of the size and contact angle recently demonstrated to be the minimum free energy path for ice nucleation and growth out of pores surrounded by silica, ${ }^{46}$ a phase transition that has the same geometry as the nucleation of gas bubbles on nanoelectrodes, although it is driven by a decrease in temperature instead of a chemical reaction.

We conclude that the increase in the contact angle with the size of the stationary bubble) originates in the more negative $\Delta \gamma_{\text {bind }}$ of the gas bubble to the electrode than to the amorphous silica. This interpretation is also consistent with the lower contact angle of the critical-sized bubble on the same electrode (Figure 3b and Movies S1 and S2), as the stronger attraction of silica for water than for gas on the model (see Methods) increases the contact angle of the bubble as it tries to advance into the silica region. ${ }^{46}$ We predict that if either the 
electrode or the surrounding silica were modified to have the same wetting properties toward both gas and water, the surface bubble would not be pinned and a constant current stationary state would not be achieved in the electrochemical experiments.

2.3. Surface Nanobubbles Must Be Nucleated Heterogeneously. According to Classical Nucleation Theory (CNT), the barrier for bubble nucleation is controlled by both the supersaturation of the gas and the free energy cost of the interface of the bubble. ${ }^{50,51}$ A surface that is more attractive to the gas than to water can decrease this barrier, facilitating the nucleation at lower supersaturations. The binding free energy per unit area of the bubble nucleus to the electrode is given by $\Delta \gamma_{\text {bind }}=\gamma_{\text {electrode-gas }}-\left(\gamma_{\text {electrode-solution }}+\gamma_{\text {solution-gas }}\right)$, where each $\gamma$ is the surface tension of the interface indicated. CNT predicts that if $\Delta \gamma_{\text {bind }}$ is negative, the critical bubble is more stable at the electrode than in solution, and its nucleation is heterogeneous. $^{52,53}$ A positive $\Delta \gamma_{\text {bind }}$ implies not only that the nucleation would proceed homogeneously, but also that the attachment of the bubble to the electrode is thermodynamically unfavorable. ${ }^{54}$ From this, we predict that homogeneously nucleated nanobubbles will not bind to the electrode and, therefore, will not lead to stationary states.

We test this prediction with molecular simulations in which we decrease $\gamma_{\text {electrode-gas }}$ and $\gamma_{\text {electrode-solution }}$ by increasing the strength $\varepsilon$ of the water-electrode and gas-electrode attraction potentials, ${ }^{55-57}$ keeping all the other interactions as indicated in Methods. Figure 7 and 8 show that the nucleation of the bubble is homogeneous when the water-electrode interactions

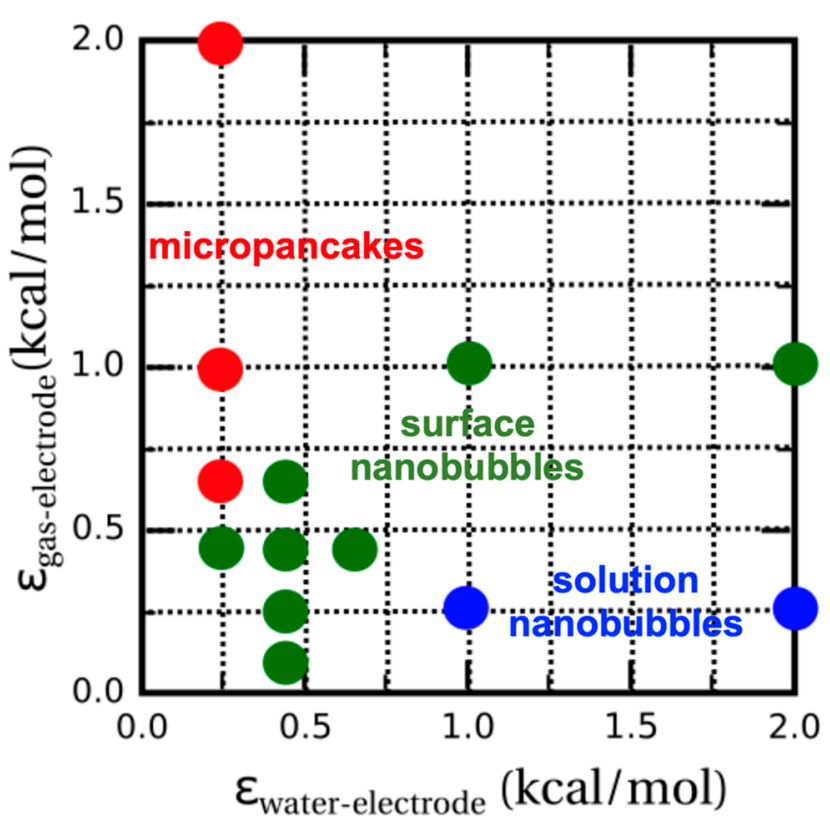

Figure 7. Competition between binding of water and gas to the electrode determines the mechanism of nucleation and whether it results in a solution bubble, surface nanobubble or one-layer-thick micropancake. The higher the attraction $\varepsilon$ of electrode for water compared to the attraction of the electrode for gas, the least favorable is the binding free energy per unit area of the bubble to the electrode, $\Delta \gamma_{\text {bind }}$. This results in the heterogeneous nucleation of micropancakes (red circles) when $\Delta \gamma_{\text {bind }}<-2 \gamma_{\text {solution-gas, }}$ heterogeneous nucleation of surface nanobubbles (green circles) when $-2 \gamma_{\text {solution-gas }}<\Delta \gamma_{\text {bind }}<$ 0 , or homogeneous nucleation of solution nanobubbles (blue circles) when $\Delta \gamma_{\text {bind }}>0$.

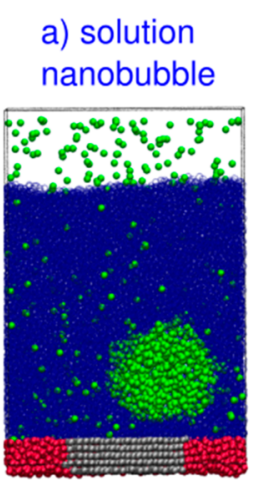

b) surface nanobubble

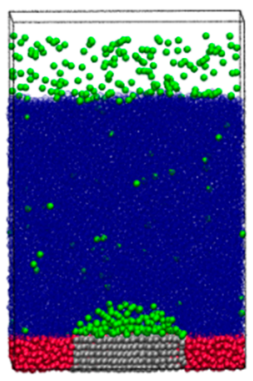

c) micropancake

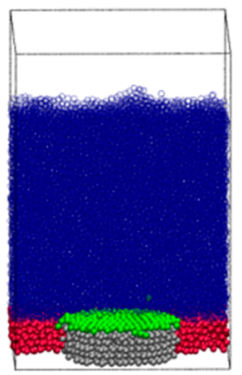

Figure 8. Snapshots of simulation cells with electrochemically generated nanobubbles in the three different regimes identified in Figure 7 . The color of the particles in the simulation cell is the same as in Figure 3. (a) Solution nanobubble nucleated homogeneously close to the electrode does not binds to the surface and does not block the electrochemical reaction, (b) nanobubble nucleated heterogeneously at the electrode remains attached to the electrode, blocking the electrochemical reaction, and (c) micropancake nucleated heterogeneously without supersaturation tightly binds to the electrode, leading to blocking and low residual currents.

are strong (i.e., $\gamma_{\text {electrode-solution }}$ is small) and the gas-electrode interactions are weak (i.e., $\gamma_{\text {electrode-gas }}$ is large), which corresponds to $\Delta \gamma_{\text {bind }}>0$. Under these conditions, the bubble nucleates just above the electrode, where the supersaturation is largest, but it never binds to it (Figure 8a and Movie S3), in agreement with the expectations from CNT. We conclude that surfaces that are unable to promote the heterogeneous nucleation of the bubble would result in a continuous increase of the current with electrochemical driving force until the diffusion-limited current is achieved, because the homogeneously nucleated bubble will not bind to and block the electrode.

We find a transition between homogeneous and heterogeneous bubble nucleation with increasing strength of gaselectrode to water-electrode attraction (Figure 7). This is consistent with a previous simulation study of Lohse and coworkers on the nucleation of nanobubbles by solvent exchange in a system of Lennard-Jones particles, where they found that bubble nucleation proceeded in solution at high solvophilicity of the surface, and at the surface for lower solvophilicity of the surface. $^{32}$ Our simulations show that the stronger is the attraction of the electrode to the gas and the weaker it is to water, the faster is the nucleation for a given driving force and the lower is the contact angle that both the critical and stationary droplets make with the electrode.

Interestingly, we find two distinct regimes for the heterogeneous nucleation of gas bubbles, which correspond to the formation of spherical cap nanobubbles (which we here call nanobubbles, Figure $8 \mathrm{~b}$ and Movies S1 and S2) and flat, one-molecule-thick nanobubbles (Figure 8c and Movies S4 and S5), which we call micropancakes and could be related to the micropancake surface nanobubbles previously described in the literature. ${ }^{58}$ The simulations indicate that flat micropancakes only occur when the electrode-gas attraction is much stronger than the electrode-water attraction (red circles in Figure 7). Experiments indicate that nanobubbles and micropancakes can be obtained for the same surface changing the supersaturation and temperature of the system, ${ }^{58-60}$ with micropancakes reported to be more stable at higher temperatures and supersaturations. In the present study, the one-layer 
micropancakes are obtained by changing the interactions of the electrode with the gas and solution, and not the nucleation conditions. Further investigation of the influence of temperature and supersaturation on the structure of the stationary bubbles would be needed to establish whether the one-layer micropancakes we find for hydrophobic surfaces are related to the micropancakes previously identified in experiments.

Both nanobubbles and micropancakes block the electrode and result in stationary states with small residual currents. We find that the residual currents of the one-layer micropancakes are lower than those of nanobubbles. We attribute this to the higher density of the micropancake, which depresses the capillary fluctuations of the gas-solution-electrode contact line needed to allow the reactants to reach the electrode. For example, the average number of gas molecules within $0.55 \mathrm{~nm}$ of the electrode is 235 for the micropancake of Figure $8 \mathrm{c}$ and 105 for the nanobubble of Figure $8 \mathrm{~b}$. The difference in the current is not as pronounced to be used as an experimental probe to distinguish one type of surface bubble from the other. The one-layer micropancakes and the spherical cap nanobubbles, however, can be distinguished by the supersaturation required for their formation. Our analysis indicates that micropancakes nucleate at the electrode without supersaturation of gas in the adjacent solution: the gas molecules generated on the bare electrode attach to it, growing a flat layer without any activation barrier. Nucleation of surface nanobubbles without supersaturation has been previously reported in experimental studies. ${ }^{60}$ According to Classical Nucleation Theory, the almost barrierless formation of the micropancakes would require that the cost of the electrode-solution interface be higher than the sum of the electrode-gas plus gas-solution interfaces, $\gamma_{\text {electrode-solution }}>\gamma_{\text {electrode-gas }}+\gamma_{\text {solution-gas, }}$ which is equivalent to $\Delta \gamma_{\text {bind }}<-2 \gamma_{\text {solution-gas. }}$. This is the condition for prewetting of the surface-liquid interface by the gas bubble. $^{61}$

The simulations indicate that a stationary surface nanobubble can be reversibly transformed into a one-layer-thick micropancake or into a solution bubble by modulation of the strength of gas-electrode and water-electrode attraction, consistent with the regions identified in Figure 7. The surface nanobubble detaches from the electrode, becoming a solution nanobubble when the water-electrode attraction becomes large compared to the gas-electrode attraction (Movie S6). If, on the other hand, the gas-electrode attraction is increased, then the nanobubble reduces its contact angle and volume and transitions from spherical cap nanobubble to a tightly bound gas monolayer. The latter transition is reversible upon a change in the interaction potential at constant driving force. The reversibility of the transitions between the different type of nanobubbles supports that the stationary states obtained in the simulations correspond to the thermodynamically stable nonequilibrium nanobubbles for the magnitude of the reaction driving force and the wetting properties of the electrode toward both water and gas.

\section{CONCLUSIONS}

In this study, we use molecular simulations to investigate the electrochemical generation and stationary states of single gas bubbles on nanoelectrodes. The effect of the electrochemical reaction is mimicked with an algorithm that creates gas molecules at the surface of the electrode and which reproduces the relation between current and reaction driving force obtained in experiments. The simulations indicate that while multiple subcritical gas nuclei can coexist on the electrode surface in the induction period before bubble formation, the critical nucleus for the formation of the nanobubbles is a single, dense cluster of gas bound to the electrode and that grows through addition of gas molecules. The size and contact angle of the critical bubble nuclei in the simulations with a Pt-like electrode are in excellent agreement with those for $\mathrm{N}_{2}$ in experiments. ${ }^{26}$ We do not find evidence for a mechanism of a nucleation based on coalescence of tiny bubbles. We conclude that the nucleation of electrochemically generated surface nanobubbles follows a classical heterogeneous pathway.

The simulations indicate that the current is largest in the induction period before the bubble nucleates at the nanoelectrode, in agreement with the results of the electrochemical experiments. ${ }^{15,16,18,25}$ After nucleation takes place, the current decreases as the bubble grows and blocks most of the reactive area, reaching a stationary state with a small residual current. The simulations show that the steady state nanobubble blocks most of the accessible area of the electrode, yet the reaction proceeds in a fringe region of $\sim 1 \AA$ width along the gaselectrode-solution interface. The stationary state is attained when the gas produced in this narrow region compensates for the loss of gas through the surface of the bubble. In agreement with the experiments, ${ }^{15,16,18,25}$ the molecular simulations predict that the residual current of the stationary state is independent of the reaction driving force, provided that this remains above a threshold value. The stationary state cannot be sustained when the driving force or the applied potential becomes too low, resulting in the dissolution of the surface nanobubble.

We identify three regimes for the nucleation of nanobubbles, depending on the sign and magnitude of the binding free energy per unit area of the gas bubble to the electrode, $\Delta \gamma_{\text {bind }}$. If the cost of the gas-electrode interface is lower than the sum of the costs of the water-electrode and water-gas interfaces, then $\Delta \gamma_{\text {bind }}$ is negative and the bubble nucleates heterogeneously at the electrode. We predict that if the electrode were very hydrophilic (or its attraction to the gas weak), the displacement of water to form the bubble on the surface would be unfavorable (i.e., $\Delta \gamma_{\text {bind }}>0$ ) and the nucleation of the bubble would proceed through a homogeneous mechanism. In that case, the simulations show that the bubble nucleates close to the electrode, because the supersaturation there is the highest, but it does not bind to its surface: it grows into a solution nanobubble. In that scenario, the binding of the bubble is unfavorable, the electrode is not blocked and the current does not drop to a residual value upon bubble nucleation. We thus conclude that all stationary electrochemically generated nanobubbles must be nucleated heterogeneously.

We find that heterogeneous nucleation at the electrode can result in hemispherical cap nanobubbles or one-layer-thick micropancakes, depending on how negative is the binding free energy of the bubble to the surface, $\Delta \gamma_{\text {bind }}$. Both hemispherical cap nanobubbles and one-layer-thick micropancakes block the electrode, leading to stationary states with residual currents. The simulations indicate that the current is lower for the micropancakes, probably due to a tighter packing of molecules of gas at the electrode surface. Nucleation theory predicts that the heterogeneous nucleation mechanism results in spherical cap nanobubbles when $0>\Delta \gamma_{\text {bind }}>-2 \gamma_{\text {gas-solution, }}$ and onemolecule-thick micropancakes when $\Delta \gamma_{\text {bind }}<-2$ $\gamma_{\text {gas-solution }}{ }^{52,53}$ The latter condition may be achieved with 
Table 1. Parameters for the Two-Body Stillinger-Weber Potentials in the Standard Simulations ${ }^{a}$

$\begin{array}{clllllllll} & \mathrm{GG}^{b} & \mathrm{WG} & \mathrm{WS} & \mathrm{WE} & \mathrm{GE} & \mathrm{GS} & \mathrm{SE}^{d} & \mathrm{EE}^{d} & \mathrm{SS}^{d} \\ \varepsilon & 0.14 & 0.18 & 0.55 & 0.45^{c} & 0.45^{c} & 0.45 & 6.189 & 6.189 & 6.189 \\ \sigma & 4.08 & 4.0 & 3.56 & 3.56 & 4.08 & 4.08 & 3.56 & 3.56 & 3.56\end{array}$

${ }^{a} \mathrm{G}$ represents the gas molecule, $\mathrm{E}$ the electrode particles, $\mathrm{W}$ water, and $\mathrm{S}$ the particles of the amorphous silica wall. Depth of the attraction well $\varepsilon$ in $\mathrm{kcal} / \mathrm{mol}$ and characteristic size $\sigma$ in $\AA$. The other parameters of the potential are same as in ref 78, $A=7.049556, B=0.6022246, a=1.8, p=4, q$ $=0 .{ }^{b}$ From ref $47 .{ }^{c}$ These values are modified in section 2.3 of Results. ${ }^{d}$ The positions of A and E are further restrained with harmonic springs.

hydrophobic electrodes and corresponds to the prewetting of gas by the surface, i.e., the formation of a coating of gas molecules at the electrode even before the concentration at that surface reaches supersaturation. The drop of the current to a residual stationary value before the concentration of gas at the electrode has reached supersaturation could be used to identify the formation of one-layer-thick micropancakes in electrochemical experiments.

The molecular simulations reveal an unanticipated dependence of the size and shape of the steady state of electrochemically generated nanobubbles on the driving force for the formation of gas. We find that the stronger the driving force for the electrochemical reaction, the larger is the stationary bubble and the higher its contact angle with the electrode. This contrasts with the independence of the residual stationary currents to the driving force. Our results indicate that there is an ensemble of nanobubble shapes associated with the same current, which suggests the existence of an attractor in these driven nonequilibrium systems. The origin of this attractor, as well as the elucidation of the dependence of the size and contact angle of stationary nanobubbles with the magnitude of the reaction driving force, are important questions that warrant further study.

To our knowledge, this study is first to model at a molecular level the nucleation, growth, and stationary states of electrochemically generated gas bubbles. Our validation that the computational methodology of this study reproduces the known phenomenology of nucleation and stationary states of nonequilibrium nanobubbles as a function of the driving force for the gas-generating reaction, combined with the computational efficiency of the coarse-grained models of this study, make the approach of this study ideal for the investigation of electrochemical reactions involving the production and consumption of gases in complex geometries, such as the arrays of nanoelectrodes used in fuel cells, ${ }^{62}$ to understand how the distribution of nanocatalysts, their activity and the nature of their supporting medium impacts the nucleation, size, and coalescence of gas bubbles, ${ }^{5}$ as well as to elucidate what is the smallest size of electrode that can sustain the production of surface and solution nanobubbles, ${ }^{14,53,63}$ and how structural and chemical heterogeneities in the electrode impact the rate of formation of bubbles.

We have assumed in our simulations that the electrode is a perfect disk, and the interactions with the reactant are inhomogeneous only at its boundary with the amorphous silica. Even for that idealized scenario, mass transport limitations due to the electrode geometry would result in non uniform currents in experiments, ${ }^{64}$ and a radial profile of supersaturation of gas over the electrode. In that case, bubble nucleation would occur in the region of higher supersaturation, and care should be taken to consider the maximal, not the average, supersaturation when deriving contact angles from current-voltage relations in experiments. Moreover, electrodes used in gas-generation experiments can display structural and chemical heterogeneities in a wide range of length-scales. As the critical nuclei of gas bubbles are nanoscopic, even heterogeneities on that length-scale could dramatically increase the nucleation rate by locally increasing gas supersaturation in the immediacy of reaction hot-spots and by providing defects or regions with stronger affinity for the gas that stabilize the bubble nucleus and decrease the nucleation barrier. Pores and crevices, for example, have been shown to increase nucleation rates dramatically, ${ }^{46,65-68}$ even allowing nucleation without supersaturation. ${ }^{69}$ The synergy between supersaturation, and increased stabilization of gas nuclei can yield sites with nucleation rates several orders of magnitude faster than the average, and result in variability of current-voltage relations for different electrodes of the same material. ${ }^{26,70}$ The synergism between the modeling approach of this study and experimental methods that can map the electrochemistry and topography at the nanoscale $e^{71-73}$ opens the doors for an unprecedented characterization and control of the formation of gas bubbles at the nanoscale, a key step for improving the design and performance of electrocatalysts for reactions involving gases.

\section{METHODS}

4.1. Model. The simulation box, illustrated in Figure 1, contains initially three regions distributed along the direction perpendicular to the electrode surface: a solid slab, an aqueous solution, and an empty reservoir to collect the excess gas generated in the simulation. The cell overall dimensions are $100 \AA \times 100 \AA \times 150 \AA$ and is periodic in the three directions. The $9 \AA$ wide solid slab is in contact with an aqueous phase with 33400 water molecules that initially extends over other $100 \AA$, followed by an empty reservoir $41 \AA$ wide. The solid consists of an amorphous silica matrix modeled as in refs 46, 74-77 surrounding a cylindrical electrode of $25 \AA$ radius, which consists of a Pt-like $f c c$ lattice with unit cell size $5 \AA^{69}$ spacing, that exposes its [001] face to the fluid.

4.2. Force Field. Interactions between all particles are modeled with the functional form of the Stillinger-Weber (SW) potential. ${ }^{78}$ Water molecules are represented with the monatomic water model $\mathrm{mW},{ }^{45}$ which reproduces the structure, ${ }^{45,79-81}$ anomalies, ${ }^{45,79,82}$ and phase behavior ${ }^{63,79,83-89}$ of water and its interfaces, ${ }^{52,54,90-96}$ including hydrophobic ${ }^{47,48,57,97-99}$ and ionic ${ }^{100-103}$ interactions, at less than $1 \%$ of the cost of all atom water models. ${ }^{45,56}$ The interactions between water and the particles in the solid slab that contains the electrode and the amorphous silica, and between the gas and the particles in the slab, are represented with two-body SW potentials. The initial parameters for the interaction between water and the electrode (WE) and the gas and electrode (GE), listed in Table 1, are selected to be equal and corresponding to a mildly attractive interaction. The values of $\mathrm{WE}$ and GE interaction parameters are modified in section 2.3 of Results (see sampled values in Figure 7) to explore their effect on the mechanism of nucleation of the nanobubbles. In all cases, particles within the amorphous silica wall and the crystalline electrode interact with their first neighbors through soft harmonic potentials with $k=10 \mathrm{kcal} \mathrm{mol}^{-1} \AA^{-2}$ that preserve the overall structure of the electrode and the inert wall, while allowing for vibrations around the corresponding equilibrium positions. In this way, all interactions are represented by pair 
potentials, except those between water molecules, which include three-body interactions to mimic hydrogen bonding.

4.3. Simulation Method and Gas-Generation Algorithm. Molecular dynamics simulations are performed with a modified version of the open source code LAMMPS. ${ }^{104}$ The equations of motion are integrated with the velocity Verlet algorithm with a time step of $5 \mathrm{fs}$. The temperature is set to $298 \mathrm{~K}$, controlled with the Nosé-Hoover thermostat with a relaxation time of 0.25 ps. The electrochemical production of gas at the electrode is mimicked by inserting gas molecules at a random position within a disk with the same area of the electrode and $1.5 \AA$ above the plane of the Pt-like atoms. The gas molecules can only be created if there is at least one water molecule within $4 \AA$ of the creation point, because water is needed for the electrochemical reaction to proceed in the experiment. If that requirement is not met, a new gas creation attempt elsewhere above the electrode plane is carried out for the same configuration, up to ten trials. The frequency $F$ of gas creation attempts is constant in each simulation. High values of $F$ produce a response analogous to the application of a high electrochemical driving force to the electrode. The simulation cell is pre-equilibrated for at least $1 \mathrm{~ns}$ before the gasgeneration algorithm is turned on. Production runs are collected for times that range up to $100 \mathrm{~ns}$. The properties of the stationary states are computed over simulations that span 40 to $80 \mathrm{~ns}$

4.4. Analysis. The current is computed as the number of moles of gas created per time, multiplied by the Faraday constant, i.e., we assume that the production of each gas molecule involves the transfer of one electron. This number was chosen arbitrarily, and only affects the magnitude of the current, but not its trends. The error bars are computed as the standard deviation over the block averages of the current computed every 5 ns over 40 ns.

The size of the gas clusters is calculated using a clustering algorithm that connects gas molecules within $5 \AA$, to account for all first gas-gas neighbors, as revealed by the gas-gas radial distribution function of the nascent bubble. A lower bound of the size of the critical gas nucleus size was estimated by the largest gas clusters in the induction period for the formation of the bubble. The width of the region at the bubble-solution-electrode-silica interface is estimated from the half-height of the distribution of the insertion sites, collected over 40 ns simulations with $F=0.1 \mathrm{ps}^{-1}$ and accounting for all successful events of creation of a gas molecule.

The density profiles of the stationary nanobubbles reported in Figure 6 are computed from simulations over $40 \mathrm{~ns}$ of the surface nanobubbles on the $5 \mathrm{~nm}$ diameter Pt-like electrodes in the stationary state with the interactions of Table 1 . The positions of the gas molecules in the bubble are sampled every $50 \mathrm{ps}$. The boundary of the bubble is defined as the locus where the average density of gas of the stationary bubble drops to half the value from its value at the center of the bubble. The density of the stationary bubbles drop from $90 \%$ to $10 \%$ of the value at the center over about $5 \AA$.

Density map of gas-creation events in stationary nanobubbles are reported in Figure 4. The density of events is averaged during $40 \mathrm{~ns}$ evolution of the stationary state of a $5 \mathrm{~nm}$ diameter electrode in which the reaction proceeds with driving force $F=0.1 \mathrm{ps}^{-1}$, and the interactions are those of Table 1. The map of Figure 4 samples 462 gas-creation events.

For the study of the mechanisms of nucleation of nanobubbles as a function of the strength of the water-electrode and gas-electrode interaction, reported in Figure 7, molecular simulations are performed for at least $40 \mathrm{~ns}$ with driving force $F=0.2 \mathrm{ps}^{-1}$. We study the transformation from surface nanobubble to micropancake; we change the interaction potentials between gas and electrode $\varepsilon_{\mathrm{GE}}$ and water and electrode $\varepsilon_{\mathrm{WE}}$ from 0.45 and $0.45 \mathrm{kcal} \mathrm{mol}^{-1}$ to 1.0 and $0.25 \mathrm{kcal}$ $\mathrm{mol}^{-1}$, and back to check the reversibility of the transformation. We study the transformation from surface nanobubble to solution bubble by changing $\varepsilon_{\mathrm{GE}}$ and $\varepsilon_{\mathrm{WE}}$ from 0.45 and $0.25 \mathrm{kcal} \mathrm{mol}^{-1}$ to 0.25 and 1 $\mathrm{kcal} \mathrm{mol}{ }^{-1}$.

\section{ASSOCIATED CONTENT}

\section{Supporting Information}

The Supporting Information is available free of charge on the ACS Publications website at DOI: 10.1021/jacs.9b04479.

Movie S1: overhead view of formation and stationary state of a nanobubble under the conditions of Figure 2 (MPG)

Movie S2: side view of formation and stationary state of a nanobubble under the conditions of Figure 2 (MPG)

Movie S3: homogeneous nucleation and stationary state of a solution nanobubble (MPG)

Movie S4: overhead view of nucleation and stationary state of one-layer micropancake (MPG)

Movie S5: side view of nucleation and stationary state of one-layer micropancake (MPG)

Movie 6: transformation of a surface nanobubble into a solution nanobubble upon a change in the wetting properties of the electrode (MPG)

\section{AUTHOR INFORMATION}

\section{Corresponding Authors}

*Valeria.Molinero@utah.edu

*yperezsirkin@gmail.com

ORCID ${ }^{\circ}$

Damian A. Scherlis: 0000-0002-0588-287X

Valeria Molinero: 0000-0002-8577-4675

Notes

The authors declare no competing financial interest.

\section{ACKNOWLEDGMENTS}

We gratefully acknowledge insightful comments and discussions with Baron Peters, Martin Edwards, Henry S. White and Hang Ren. This study was supported by the Fulbright Foundation in the form of a fellowship to Y.A.P.S., The University of Utah through the Seed Grant "Molecular Modeling of Electrochemically Generated Nanobubbles" to V.M., and the Agencia Nacional de Promoción Científica y Tecnológica de Argentina (ANPCyT) through PICT 20163167 to D.A.S. We thank the Center of High Performance Computing at The University of Utah for technical support and a grant of computing time.

\section{REFERENCES}

(1) Zhao, X.; Ren, H.; Luo, L. Gas Bubbles in Electrochemical Gas Evolution Reactions. Langmuir 2019, 35, 5392-5408.

(2) Roger, I.; Shipman, M. A.; Symes, M. D. Earth-Abundant Catalysts for Electrochemical and Photoelectrochemical Water Splitting. Nature Rev. Chem. 2017, 1, No. 0003.

(3) Anantharaj, S.; Ede, S. R.; Sakthikumar, K.; Karthick, K.; Mishra, S.; Kundu, S. Recent Trends and Perspectives in Electrochemical Water Splitting with an Emphasis on Sulfide, Selenide, and Phosphide Catalysts of $\mathrm{Fe}, \mathrm{Co}$, and Ni: A Review. ACS Catal. 2016, 6, 80698097.

(4) Voiry, D.; Shin, H. S.; Loh, K. P.; Chhowalla, M. LowDimensional Catalysts for Hydrogen Evolution and Co 2 Reduction. Nature Rev. Chem. 2018, 2, No. 0105.

(5) Zhang, L.; Zhang, Y.; Zhang, X.; Li, Z.; Shen, G.; Ye, M.; Fan, C.; Fang, H.; Hu, J. Electrochemically Controlled Formation and Growth of Hydrogen Nanobubbles. Langmuir 2006, 22, 8109-8113.

(6) Li, Y.; Gao, W.; Ci, L.; Wang, C.; Ajayan, P. M. Catalytic Performance of Pt Nanoparticles on Reduced Graphene Oxide for Methanol Electro-Oxidation. Carbon 2010, 48, 1124-1130. 
(7) Abdel Rahim, M. A.; Abdel Hameed, R. M.; Khalil, M. W. Nickel as a Catalyst for the Electro-Oxidation of Methanol in Alkaline Medium. J. Power Sources 2004, 134, 160-169.

(8) Karlsson, R. K.; Cornell, A. Selectivity between Oxygen and Chlorine Evolution in the Chlor-Alkali and Chlorate Processes. Chem. Rev. 2016, 116, 2982-3028.

(9) Mazloomi, S.; Sulaiman, N. Influencing Factors of Water Electrolysis Electrical Efficiency. Renewable Sustainable Energy Rev. 2012, 16, 4257-4263.

(10) Mao, S.; Wen, Z.; Huang, T.; Hou, Y.; Chen, J. HighPerformance Bi-Functional Electrocatalysts of 3d Crumpled Graphene-Cobalt Oxide Nanohybrids for Oxygen Reduction and Evolution Reactions. Energy Environ. Sci. 2014, 7, 609-616.

(11) Jaramillo, T. F.; Jørgensen, K. P.; Bonde, J.; Nielsen, J. H.; Horch, S.; Chorkendorff, I. Identification of Active Edge Sites for Electrochemical H2 Evolution from Mos2 Nanocatalysts. Science 2007, 317, 100-102.

(12) Gupta, S.; Patel, N.; Fernandes, R.; Kadrekar, R.; Dashora, A.; Yadav, A.; Bhattacharyya, D.; Jha, S.; Miotello, A.; Kothari, D. Co$\mathrm{Ni}-\mathrm{B}$ Nanocatalyst for Efficient Hydrogen Evolution Reaction in Wide Ph Range. Appl. Catal., B 2016, 192, 126-133.

(13) Kaden, W. E.; Wu, T.; Kunkel, W. A.; Anderson, S. L. Electronic Structure Controls Reactivity of Size-Selected Pd Clusters Adsorbed on Tio2 Surfaces. Science 2009, 326, 826-829.

(14) Li, S.; Du, Y.; He, T.; Shen, Y.; Bai, C.; Ning, F.; Hu, X.; Wang, W.; Xi, S.; Zhou, X. Nanobubbles: An Effective Way to Study GasGenerating Catalysis on a Single Nanoparticle. J. Am. Chem. Soc. 2017, 139, 14277-14284.

(15) Luo, L.; White, H. S. Electrogeneration of Single Nanobubbles at Sub-50-Nm-Radius Platinum Nanodisk Electrodes. Langmuir 2013, 29, 11169-11175.

(16) Chen, Q.; Luo, L.; White, H. S. Electrochemical Generation of a Hydrogen Bubble at a Recessed Platinum Nanopore Electrode. Langmuir 2015, 31, 4573-4581.

(17) German, S. R.; Edwards, M. A.; Chen, Q.; Liu, Y.; Luo, L.; White, H. S. Electrochemistry of Single Nanobubbles. Estimating the Critical Size of Bubble-Forming Nuclei for Gas-Evolving Electrode Reactions. Faraday Discuss. 2016, 193, 223-240.

(18) Chen, Q.; Luo, L.; Faraji, H.; Feldberg, S. W.; White, H. S. Electrochemical Measurements of Single H2 Nanobubble Nucleation and Stability at Pt Nanoelectrodes. J. Phys. Chem. Lett. 2014, 5, 35393544.

(19) Chen, Q.; Wiedenroth, H. S.; German, S. R.; White, H. S. Electrochemical Nucleation of Stable N2 Nanobubbles at $\mathrm{Pt}$ Nanoelectrodes. J. Am. Chem. Soc. 2015, 137, 12064-12069.

(20) German, S. R.; Chen, Q.; Edwards, M. A.; White, H. S. Electrochemical Measurement of Hydrogen and Nitrogen Nanobubble Lifetimes at Pt Nanoelectrodes. J. Electrochem. Soc. 2016, 163, H3160-H3166.

(21) German, S. R.; Edwards, M. A.; Chen, Q.; White, H. S. Laplace Pressure of Individual H 2nanobubbles from Pressure-Addition Electrochemistry. Nano Lett. 2016, 16, 6691-6694.

(22) German, S. R.; Edwards, M. A.; Ren, H.; White, H. S. Critical Nuclei Size, Rate, and Activation Energy of H2 Gas Nucleation. J. Am. Chem. Soc. 2018, 140, 4047-4053.

(23) Liu, Y.; Edwards, M. A.; German, S. R.; Chen, Q.; White, H. S. The Dynamic Steady State of an Electrochemically Generated Nanobubble. Langmuir 2017, 33, 1845-1853.

(24) Moreno-Soto, Á.; German, S. R.; Ren, H.; Van Der Meer, D.; Lohse, D.; Edwards, M. A.; White, H. S. The Nucleation Rate of Single O_2 Nanobubbles at Pt Nanoelectrodes. Langmuir 2018, 34, 7309-7318.

(25) Ren, H.; German, S. R.; Edwards, M. A.; Chen, Q.; White, H. S. Electrochemical Generation of Individual O2 Nanobubbles Via $\mathrm{H} 2 \mathrm{o} 2$ Oxidation. J. Phys. Chem. Lett. 2017, 8, 2450-2454.

(26) Edwards, M. A.; White, H. S.; Ren, H. Voltammetric Determination of the Stochastic Formation Rate and Geometry of Individual H2, N2 and O2 Bubble Nuclei. ACS Nano 2019, DOI: 10.1021 /acsnano.9b01015.
(27) Hui, F.; Li, B.; He, P.; Hu, J.; Fang, Y. Electrochemical Fabrication of Nanoporous Polypyrrole Film on Hopg Using Nanobubbles as Templates. Electrochem. Commun. 2009, 11, 639642 .

(28) Chen, Q.; Luo, L. Correlation between Gas Bubble Formation and Hydrogen Evolution Reaction Kinetics at Nanoelectrodes. Langmuir 2018, 34, 4554-4559.

(29) Chen, Q.; Ranaweera, R.; Luo, L. Hydrogen Bubble Formation at Hydrogen-Insertion Electrodes. J. Phys. Chem. C 2018, 122, $15421-15426$.

(30) Weijs, J. H.; Snoeijer, J. H.; Lohse, D. Formation of Surface Nanobubbles and the Universality of Their Contact Angles: A Molecular Dynamics Approach. Phys. Rev. Lett. 2012, 108, 104501.

(31) Liu, Y.; Zhang, X. A Review of Recent Theoretical and Computational Studies on Pinned Surface Nanobubbles. Chin. Phys. B 2018, 27, No. 014401.

(32) Xiao, Q.; Liu, Y.; Guo, Z.; Liu, Z.; Lohse, D.; Zhang, X. Solvent Exchange Leading to Nanobubble Nucleation: A Molecular Dynamics Study. Langmuir 2017, 33, 8090-8096.

(33) Che, Z.; Theodorakis, P. E. Formation, Dissolution and Properties of Surface Nanobubbles. J. Colloid Interface Sci. 2017, 487, $123-129$.

(34) Xiao, Q.; Liu, Y.; Guo, Z.; Liu, Z.; Frenkel, D.; Dobnikar, J.; Zhang, X. What Experiments on Pinned Nanobubbles Can Tell About the Critical Nucleus for Bubble Nucleation. Eur. Phys. J. E 2017, 40, 114.

(35) Diemand, J.; Angélil, R.; Tanaka, K. K.; Tanaka, H. Direct Simulations of Homogeneous Bubble Nucleation: Agreement with Classical Nucleation Theory and No Local Hot Spots. Phys. Rev. E 2014, 90, No. 052407.

(36) Petsev, N. D.; Shell, M. S.; Leal, L. G. Dynamic Equilibrium Explanation for Nanobubbles' Unusual Temperature and Saturation Dependence. Phys. Rev. E 2013, 88, No. 010402.

(37) Knott, B. C.; LaRue, J. L.; Wodtke, A. M.; Doherty, M. F.; Peters, B. Communication: Bubbles, Crystals, and Laser-Induced Nucleation. J. Chem. Phys. 2011, 134, 171102.

(38) Torabi, K.; Corti, D. S. Toward a Molecular Theory of Homogeneous Bubble Nucleation: Ii. Calculation of the Number Density of Critical Nuclei and the Rate of Nucleation. J. Phys. Chem. B 2013, 117, 12491-12504.

(39) Wang, Z.-J.; Valeriani, C.; Frenkel, D. Homogeneous Bubble Nucleation Driven by Local Hot Spots: A Molecular Dynamics Study. J. Phys. Chem. B 2009, 113, 3776-3784.

(40) Abascal, J. L.; Gonzalez, M. A.; Aragones, J. L.; Valeriani, C. Homogeneous Bubble Nucleation in Water at Negative Pressure: A Voronoi Polyhedra Analysis. J. Chem. Phys. 2013, 138, No. 084508.

(41) Meadley, S. L.; Escobedo, F. A. Thermodynamics and Kinetics of Bubble Nucleation: Simulation Methodology. J. Chem. Phys. 2012, 137, No. 074109.

(42) Maheshwari, S.; Van Der Hoef, M.; Rodríguez Rodríguez, J.; Lohse, D. Leakiness of Pinned Neighboring Surface Nanobubbles Induced by Strong Gas-Surface Interaction. ACS Nano 2018, 12, 2603-2609.

(43) Wang, L.; Wang, X.; Wang, L.; Hu, J.; Wang, C. L.; Zhao, B.; Zhang, X.; Tai, R.; He, M.; Chen, L.; Zhang, L. Formation of Surface Nanobubbles on Nanostructured Substrates. Nanoscale 2017, 9, $1078-1086$

(44) Koishi, T.; Yoo, S.; Yasuoka, K.; Zeng, X. C.; Narumi, T.; Susukita, R.; Kawai, A.; Furusawa, H.; Suenaga, A.; Okimoto, N.; Futatsugi, N.; Ebisuzaki, T. Nanoscale Hydrophobic Interaction and Nanobubble Nucleation. Phys. Rev. Lett. 2004, 93, 185701.

(45) Molinero, V.; Moore, E. B. Water Modeled as an Intermediate Element between Carbon and Silicon. J. Phys. Chem. B 2009, 113, 4008-4016.

(46) David, R. O.; Marcolli, C.; Fahrni, J.; Qiu, Y.; Perez Sirkin, Y. A.; Molinero, V.; Mahrt, F.; Brühwiler, D.; Lohmann, U.; Kanji, Z. A. Pore Condensation and Freezing Is Responsible for Ice Formation Below Water Saturation for Porous Particles. Proc. Natl. Acad. Sci. U. S. A. 2019, 116, 8184-8189. 
(47) Knott, B. C.; Molinero, V.; Doherty, M. F.; Peters, B. Homogeneous Nucleation of Methane Hydrates: Unrealistic under Realistic Conditions. J. Am. Chem. Soc. 2012, 134, 19544-19547.

(48) Jacobson, L. C.; Molinero, V. A Methane-Water Model for Coarse-Grained Simulations of Solutions and Clathrate Hydrates. J. Phys. Chem. B 2010, 114, 7302-7311.

(49) Hynes, W. M. Handbook of Chemistry and Physics, 95th ed.; CRC, 2015.

(50) Blander, M.; Katz, J. L. Bubble Nucleation in Liquids. AIChE J. 1975, 21, 833-848.

(51) Jones, S.; Evans, G.; Galvin, K. Bubble Nucleation from Gas Cavities-a Review. Adv. Colloid Interface Sci. 1999, 80, 27-50.

(52) Qiu, Y.; Odendahl, N.; Hudait, A.; Mason, R.; Bertram, A. K.; Paesani, F.; DeMott, P. J.; Molinero, V. Ice Nucleation Efficiency of Hydroxylated Organic Surfaces Is Controlled by Their Structural Fluctuations and Mismatch to Ice. J. Am. Chem. Soc. 2017, 139, 3052-3064.

(53) Qiu, Y.; Hudait, A.; Molinero, V. How Size and Aggregation of Ice-Binding Proteins Control Their Ice Nucleation Efficiency. J. Am. Chem. Soc. 2019, 141, 7439-7452.

(54) Qiu, Y.; Molinero, V. Why Is It So Difficult to Identify the Onset of Ice Premelting? J. Phys. Chem. Lett. 2018, 9, 5179-5182.

(55) Greenwood, J. On the Dmt Theory. Tribol. Lett. 2007, 26, 203-211.

(56) Lu, J.; Qiu, Y.; Baron, R.; Molinero, V. Coarse-Graining of Tip4p/2005, Tip4p-Ew, Spc/E, and Tip3p to Monatomic Anisotropic Water Models Using Relative Entropy Minimization. J. Chem. Theory Comput. 2014, 10, 4104-4120.

(57) Qiu, Y.; Molinero, V. Strength of Alkane-Fluid Attraction Determines the Interfacial Orientation of Liquid Alkanes and Their Crystallization through Heterogeneous or Homogeneous Mechanisms. Crystals 2017, 7, 86.

(58) Zhang, X. H.; Maeda, N.; Hu, J. Thermodynamic Stability of Interfacial Gaseous States. J. Phys. Chem. B 2008, 112, 13671-13675.

(59) Seddon, J. R.; Kooij, E. S.; Poelsema, B.; Zandvliet, H. J.; Lohse, D. Surface Bubble Nucleation Stability. Phys. Rev. Lett. 2011, 106, No. 056101.

(60) Seddon, J. R.; Lohse, D. Nanobubbles and Micropancakes: Gaseous Domains on Immersed Substrates. J. Phys.: Condens. Matter 2011, 23, 133001.

(61) Bonn, D. Wetting Transitions. Curr. Opin. Colloid Interface Sci. 2001, 6, 22-27.

(62) Debe, M. K. Electrocatalyst Approaches and Challenges for Automotive Fuel Cells. Nature 2012, 486, 43.

(63) Factorovich, M. H.; Molinero, V.; Scherlis, D. A. Vapor Pressure of Water Nanodroplets. J. Am. Chem. Soc. 2014, 136, 45084514.

(64) Newman, J. Current Distribution on a Rotating Disk Below the Limiting Current. J. Electrochem. Soc. 1966, 113, 1235-1241.

(65) Marcolli, C. Deposition Nucleation Viewed as Homogeneous or Immersion Freezing in Pores and Cavities. Atmos. Chem. Phys. 2014, 14, 2071-2104.

(66) Diao, Y.; Myerson, A. S.; Hatton, T. A.; Trout, B. L. Surface Design for Controlled Crystallization: The Role of Surface Chemistry and Nanoscale Pores in Heterogeneous Nucleation. Langmuir 2011, 27, 5324-5334.

(67) Page, A. J.; Sear, R. P. Heterogeneous Nucleation in and out of Pores. Phys. Rev. Lett. 2006, 97, No. 065701.

(68) Campbell, J. M.; Meldrum, F. C.; Christenson, H. K. Observing the Formation of Ice and Organic Crystals in Active Sites. Proc. Natl. Acad. Sci. U. S. A. 2017, 114, 810-815.

(69) Kovács, T.; Meldrum, F.; Christenson, H. Crystal Nucleation without Supersaturation. J. Phys. Chem. Lett. 2012, 3, 1602-1606.

(70) Edwards, M.; Robinson, D.; Ren, H.; Cheyne, C.; Tan, C.; White, H. S. Nanoscale Electrochemical Kinetics \& Dynamics: The Challenges and Opportunities of Single-Entity Measurements. Faraday Discuss. 2018, 210, 9-28.
(71) Kai, T.; Zoski, C. G.; Bard, A. J. Scanning Electrochemical Microscopy at the Nanometer Level. Chem. Commun. 2018, 54, $1934-1947$.

(72) Ebejer, N.; Schnippering, M.; Colburn, A. W.; Edwards, M. A.; Unwin, P. R. Localized High Resolution Electrochemistry and Multifunctional Imaging: Scanning Electrochemical Cell Microscopy. Anal. Chem. 2010, 82, 9141-9145.

(73) Williams, C. G.; Edwards, M. A.; Colley, A. L.; Macpherson, J. V.; Unwin, P. R. Scanning Micropipet Contact Method for HighResolution Imaging of Electrode Surface Redox Activity. Anal. Chem. 2009, 81, 2486-2495.

(74) Moore, E. B.; de la Llave, E.; Welke, K.; Scherlis, D. A.; Molinero, V. Freezing, Melting and Structure of Ice in a Hydrophilic Nanopore. Phys. Chem. Chem. Phys. 2010, 12, 4124-4134.

(75) Moore, E. B.; Allen, J. T.; Molinero, V. Liquid-Ice Coexistence Below the Melting Temperature for Water Confined in Hydrophilic and Hydrophobic Nanopores. J. Phys. Chem. C 2012, 116, 75077514.

(76) Factorovich, M. H.; Gonzalez Solveyra, E.; Molinero, V.; Scherlis, D. A. Sorption Isotherms of Water in Nanopores: Relationship between Hydropohobicity, Adsorption Pressure, and Hysteresis. J. Phys. Chem. C 2014, 118, 16290-16300.

(77) Factorovich, M. H.; Molinero, V.; Scherlis, D. A. HydrogenBond Heterogeneity Boosts Hydrophobicity of Solid Interfaces. J. Am. Chem. Soc. 2015, 137, 10618-10623.

(78) Stillinger, F. H.; Weber, T. A. Computer Simulation of Local Order in Condensed Phases of Silicon. Phys. Rev. B: Condens. Matter Mater. Phys. 1985, 31, 5262-5271.

(79) Moore, E. B.; Molinero, V. Growing Correlation Length in Supercooled Water. J. Chem. Phys. 2009, 130, 244505.

(80) Moore, E. B.; Molinero, V. Is It Cubic? Ice Crystallization from Deeply Supercooled Water. Phys. Chem. Chem. Phys. 2011, 13, 20008-20016.

(81) Lupi, L.; Hudait, A.; Peters, B.; Grünwald, M.; Mullen, R. G.; Nguyen, A. H.; Molinero, V. Role of Stacking Disorder in Ice Nucleation. Nature 2017, 551, 218.

(82) Lu, J.; Chakravarty, C.; Molinero, V. Relationship between the Line of Density Anomaly and the Lines of Melting, Crystallization, Cavitation, and Liquid Spinodal in Coarse-Grained Water Models. J. Chem. Phys. 2016, 144, 234507.

(83) Moore, E. B.; De La Llave, E.; Welke, K.; Scherlis, D. A.; Molinero, V. Freezing, Melting and Structure of Ice in a Hydrophilic Nanopore. Phys. Chem. Chem. Phys. 2010, 12, 4124-4134.

(84) Hudait, A.; Qiu, S.; Lupi, L.; Molinero, V. Free Energy Contributions and Structural Characterization of Stacking Disordered Ices. Phys. Chem. Chem. Phys. 2016, 18, 9544-9553.

(85) Jacobson, L. C.; Hujo, W.; Molinero, V. Amorphous Precursors in the Nucleation of Clathrate Hydrates. J. Am. Chem. Soc. 2010, 132, 11806-11811.

(86) Johnston, J. C.; Molinero, V. Crystallization, Melting, and Structure of Water Nanoparticles at Atmospherically Relevant Temperatures. J. Am. Chem. Soc. 2012, 134, 6650-6659.

(87) Lupi, L.; Hudait, A.; Molinero, V. Heterogeneous Nucleation of Ice on Carbon Surfaces. J. Am. Chem. Soc. 2014, 136, 3156-3164.

(88) Hudait, A.; Molinero, V. Ice Crystallization in Ultrafine WaterSalt Aerosols: Nucleation, Ice-Solution Equilibrium, and Internal Structure. J. Am. Chem. Soc. 2014, 136, 8081-8093.

(89) Hudait, A.; Moberg, D. R.; Qiu, Y.; Odendahl, N.; Paesani, F.; Molinero, V. Preordering of Water Is Not Needed for Ice Recognition by Hyperactive Antifreeze Proteins. Proc. Natl. Acad. Sci. U. S. A. 2018, 115, 8266-8271.

(90) Lupi, L.; Kastelowitz, N.; Molinero, V. Vapor Deposition of Water on Graphitic Surfaces: Formation of Amorphous Ice, Bilayer Ice, Ice I, and Liquid Water. J. Chem. Phys. 2014, 141, 18 C508.

(91) Shepherd, T. D.; Koc, M. A.; Molinero, V. The Quasi-Liquid Layer of Ice under Conditions of Methane Clathrate Formation. J. Phys. Chem. C 2012, 116, 12172-12180. 
(92) Nguyen, A. H.; Koc, M. A.; Shepherd, T. D.; Molinero, V. Structure of the Ice-Clathrate Interface. J. Phys. Chem. C 2015, 119, 4104-4117.

(93) Qiu, Y.; Lupi, L.; Molinero, V. Is Water at the Graphite Interface Vapor-Like or Ice-Like? J. Phys. Chem. B 2018, 122, 36263634.

(94) Hudait, A.; Allen, M. T.; Molinero, V. Sink or Swim: Ions and Organics at the Ice-Air Interface. J. Am. Chem. Soc. 2017, 139, 10095-10103.

(95) Pickering, I.; Paleico, M.; Perez Sirkin, Y. A.; Scherlis, D. A.; Factorovich, M. H. Grand Canonical Investigation of the Quasi Liquid Layer of Ice: Is It Liquid? J. Phys. Chem. B 2018, 122, 48804890.

(96) Kastelowitz, N.; Molinero, V. Ice-Liquid Oscillations in Nanoconfined Water. ACS Nano 2018, 12, 8234-8239.

(97) Baron, R.; Molinero, V. Water-Driven Cavity-Ligand Binding: Comparison of Thermodynamic Signatures from Coarse-Grained and Atomic-Level Simulations. J. Chem. Theory Comput. 2012, 8, 36963704.

(98) Song, B.; Molinero, V. Thermodynamic and Structural Signatures of Water-Driven Methane-Methane Attraction in CoarseGrained Mw Water. J. Chem. Phys. 2013, 139, No. 054511.

(99) Qiu, Y.; Molinero, V. Morphology of Liquid-Liquid Phase Separated Aerosols. J. Am. Chem. Soc. 2015, 137, 10642-10651.

(100) DeMille, R. C.; Molinero, V. Coarse-Grained Ions without Charges: Reproducing the Solvation Structure of Nacl in Water Using Short-Ranged Potentials. J. Chem. Phys. 2009, 131, 034107.

(101) Lu, J.; Jacobson, L. C.; Perez Sirkin, Y. A.; Molinero, V. HighResolution Coarse-Grained Model of Hydrated Anion-Exchange Membranes That Accounts for Hydrophobic and Ionic Interactions through Short-Ranged Potentials. J. Chem. Theory Comput. 2017, 13, 245-264.

(102) Lu, J.; Miller, C.; Molinero, V. Parameterization of a CoarseGrained Model with Short-Ranged Interactions for Modeling Fuel Cell Membranes with Controlled Water Uptake. Phys. Chem. Chem. Phys. 2017, 19, 17698-17707.

(103) Perez Sirkin, Y. A.; Factorovich, M. H.; Molinero, V.; Scherlis, D. A. Vapor Pressure of Aqueous Solutions of Electrolytes Reproduced with Coarse-Grained Models without Electrostatics. J. Chem. Theory Comput. 2016, 12, 2942-2949.

(104) Plimpton, S. Fast Parallel Algorithms for Short-Range Molecular Dynamics. J. Comput. Phys. 1995, 117, 1-19. 